\title{
TSHZ3 is A Prognostic Biomarker with Immune Infiltration in Stomach Adenocarcinoma and Bladder Urothelial Carcinoma
}

\author{
Xi Zhang \\ Syndrome Laboratory of Integrated Chinese and Western Medicine,School of Chinese Medicine, Southern Medical University

\section{Ming Wang} \\ Department of Traditional Chinese Medicine,Zhujiang Hospital of Southern Medical University \\ Han-qi Lu \\ Syndrome Laboratory of Integrated Chinese and Western Medicine,School of Chinese Medicine,Southern Medical University \\ Qiu-xing He \\ Syndrome Laboratory of Integrated Chinese and Western Medicine, School of Chinese Medicine,Southern Medical University \\ Yan-ting You \\ Syndrom Laboratory of Integrated Chinese and Western Medicine,School of Chinese Medicine,Southern Medical University \\ Xing-hong Zhou \\ Syndrome Laboratory of Integrated Chinese and Western Medicine,School of Chinese Medicine,Southern Medical University \\ Xiu-qiong Fu \\ School of Chinese Medicine,Hong Kong Babtist University \\ Dong-hui Liang \\ Department of Traditional Chinese Medicine,Zhujiang Hospital of Southern Medical University \\ Hiu Yee Kwan \\ School of Chinese Medicine,Hong Kong Baptist University \\ Xiao-shan Zhao ( $\nabla$ zhaoxs0609@163.com) \\ Lin Zhou ( $\nabla$ zlecho@163.com) \\ Endocrinology Department,Nanfang Hospital,Southern Medical University
}

\section{Research}

Keywords: TSHZ3, immune infiltration, prognosis, stomach adenocarcinoma, bladder urothelial carcinoma

Posted Date: May 14th, 2020

DOI: https://doi.org/10.21203/rs.3.rs-27520/v1

License: (c) (i) This work is licensed under a Creative Commons Attribution 4.0 International License. Read Full License 


\section{Abstract}

Background: This study aimed to investigate the expressions of Teashirt zinc finger homeobox 3 (TSHZ3) in different cancer types and identify the cancers of which prognosis can be predicted by TSHZ3 expression. Furthermore, we aimed to explore the correlations between tumor-infiltrating immune cells (TIICS) and TSHZ3 expression in these cancers.

Methods: TSHZ3 expression was analyzed by the Oncomine database and Gene Expression Profiling Interactive Analysis (GEPIA). We examined the influence of TSHZ3 on the clinical prognosis with Kaplan-Meier plotter and TIMER (Tumor Immune Estimation Resource). The correlations between tumor immune infiltrates and TSHZ3 expressions were investigated with TIMER and validated by GEPIA.

Results: TSHZ3 expressions were significantly higher in stomach adenocarcinoma (STAD) and bladder urothelial carcinoma (BLCA) when compared to normal tissues. Survival analysis results showed that high TSHZ3 expression was correlated with poor overall survival (OS) in STAD and BLCA. The infiltrations of pro-tumorigenic TIICs (M2 macrophages, monocytes, B cells and pDCs) were positively correlated with TSHZ3 expression in STAD and BLCA. Besides, the infiltration of other pro-tumorigenic TIICs (tumor-associated macrophages (TAMs), Th2 cells and Tregs) and expression of T cell exhaustion markers (PD-1 and TIM-3) were positively correlated with TSHZ3 expression in STAD.

Conclusions: TSHZ3 expression was up-regulated in STAD and BLCA. High TSHZ3 expression predicted poor prognosis of patients with STAD or BLCA. In addition, TSHZ3 expression potentially contributes to the increased infiltrations of pro-tumorigenic TIICs. TSHZ3 can be used as a prognostic biomarker for predicting prognosis and immune infiltration in STAD and BLCA.

\section{Background}

Gastric cancer (GC) is the second most common cause of cancer-related deaths in the world. It has a high incidence and poor prognosis [1]. Urinary bladder cancer is the most common malignancy involving the urinary system. The bladder urothelial carcinoma (BLCA) is the most common histological type of bladder cancer, which represents more than $90 \%$ of all the bladder carcinomas cases [2,3].

Solid tumors including GC and BLCA are commonly infiltrated by immune cells. The immune infiltration strongly influences the development of GC and BLCA $[4,5]$. Modulating the immune infiltration is a promising therapeutic strategy for GC and BLCA [6, 7]. Indeed, growing evidences suggest that the tumor-infiltrating immune cells (TIICs), such as CD8 + tumor-infiltrating T cells (TITCs) and CD68 + tumor-associated macrophages (TAMs), strongly influence the prognosis and efficacy of immunotherapy and chemotherapy [8-11]. Meanwhile,it has been widely reported that different subsets of some TIICs have diverse biological effects. For example, M2 macrophages, plasmacytoid dendritic cells (pDCs), Treg cells and Th2 cells are protumorigenic while M1 macrophages, one subsets of conventional DCs (cDC1s), Tfh cells and Th1 cells are anti-tumorigenic [12, 13]. Therefore, it is important to elucidate the TIIC profile and identify novel therapeutic targets for GC and BLCA.

Teashirt zinc finger homeobox 3 (TSHZ3) is a gene encoding a zinc finger transcription factor and has been widely studied in the nervous system and the differentiation of smooth muscle cell [14-16]. Moreover, TSHZ3 also functions as tumor suppressor in brain glioma and ovarian cancer but as it is an oncogene in breast cancer [17-19]. A recent study suggests that TSHZ3 predict poor prognosis in tongue squamous cell carcinomas because it modulates the immune infiltration [20]. However, the role of TSHZ3 in other cancer growth and the underlying mechanism of action is not known. In our present study, we employed systemic biology approach, Oncomine and Gene Expression Profiling Interactive Analysis (GEPIA), to analyze the expressions of TSHZ3 in various cancers, and evaluated the correlations between TSHZ3 expression and the prognosis of malignancy patients in databases such as Kaplan-Meier plotter and Tumor Immune Estimation Resource (TIMER) [21-24]. Furthermore, we investigated the correlation of TSHZ3 and TIICs in the diverse tumor microenvironments by using TIMER. Our study not only reveals the critical role of TSHZ3 in BLCA and GC development, but also suggests a potential association and a regulatory role of TSHZ3 in TIICs.

\section{Materials And Methods}

\section{TSHZ3 Gene Expression Level Analysis}

The expression level of TSHZ3 in different types of cancers was identified via the Oncomine database (https://www. oncomine.org/resource/login.html) and the GEPIA database [22, 24]. In the Oncomine database, benign tumor data was excluded and the rest was included for pooled analysis with the Compare module, the threshold was determined by the following values: P-value of all, fold change of all, and gene ranking of all. TSHZ3 gene expression level analysis was conducted by GEPIA while the cancer types were lacking or samples were small in Oncomine. Significant statistical differences in TSHZ3 expression between tumor and normal tissues was considered to be existed while the p-value of $<0.05$.

\section{Survival Analysis}

We evaluated the effect of TSHZ3 on clinical prognosis by using Kaplan-Meier plotter and TIMER up to Jan 4th 2020. Kaplan-Meier plotter is able to evaluate the effect of special genes on survival using 13,316 cancer samples, which include 6,234 breast, 1,440 gastric, 3,452 lung and 2,190 ovarian cancer samples. The relationship between expression of TSHZ3 and survival in breast, gastric, lung and ovarian cancers was analyzed by Kaplan- 
Meier plotter (http://kmplot.com/analysis/) [21].The log-rank P-value and hazard ratio (HR) with 95\% confidence intervals and were presented. TIMER (https://cistrome.shinyapps.io/timer/) is a web server that includes 10,897 cancers samples from The Cancer Genome Atlas (TCGA). We carried out the survival analysis of different cancer types with the Survival module of TIMER. Results from both cox proportional hazard model method and KaplanMeier method were included for further evaluation.Database with larger sample number was selected to present Kaplan-Meier plot to increase the credibility of the results.

\section{Immune Infiltration Analysis}

TIMER is a comprehensive web resource for systematic evaluation of immune infiltrations across different cancer types [23], which infers the abundance of TIICs from gene expression profiles by applying a deconvolution published statistical method [25]. Thus, we roughly evaluated the immune infiltrates abundance, including B cell, CD8 + T cell, CD4 + T cell, dendritic cell, neutrophil and macrophage by the Gene module at first. Next, we further confirmed the correlations between TSHZ3 expression and TIICs and its' subsets with gene markers by using the Correlation module of TIMER. These immune gene markers are commonly referenced in recent studies [12,26, 27]. Gene expression levels were adjusted by tumor purity [28] Except for the exhausted T cell, we regarded correlations between TSHZ3 expression and TIICs as significant in the Correlation module only when all the correlations of the TIIC gene markers were consistent and significant. Significant correlations revealed by TIMER were furthered validated for the given sets of TCGA data via GEPIA. The correlation coefficient was determined by Spearman method. The X-axis presented the TSHZ3 expression, and Y-axis presented the gene markers of the immune cells. The datasets of cancer and normal tissues were used for analysis.

\section{Statistical Analysis}

All analyses were performed using the independent sample t-test or Spearman correlation analysis, as appropriate. Survival analysis in TIMER were conducted with the cox proportional hazard model method and Kaplan-Meier method. Survival curves were produced via the Kaplan-Meier method and the log rank test. P-values $<0.05$ were considered statistically significant.

\section{Results}

\section{The Expression Levels of TSHZ3 in Different Human Cancer Types}

To investigate the differences of TSHZ3 expressions in cancer and normal tissues, TSHZ3 expression levels in cancers and related normal tissues were evaluated with the Oncomine database and GEPIA. The analysis revealed that TSHZ3 expressions were higher in 14 cancer types or subtypes when compared with the normal tissues. These cancers include bladder urothelial carcinoma (BLCA), cervical cancer, gastric cancer, stomach adenocarcinoma (STAD), liver cancer, lung cancer, lung squamous cell carcinoma (LUSC), melanoma, myeloma (multiple myeloma), skin cancer, ovarian serous cystadenocarcinoma (OV), pancreatic cancer, pancreatic adenocarcinoma (PAAD), and pheochromocytoma and paraganglioma (PCPG). However, expressions of TSHZ3 were lower in adrenal cortex carcinoma (ACC), breast invasive carcinoma (BRCA), leukemia, ovarian cancer, prostate cancer, prostate adenocarcinoma (PRAD), uterine corpus endometrial carcinoma (UCEC) and uterine carcinosarcoma (UCS) compared with normal tissues (Table 1, Fig. 1).The details of TSHZ3expression in different cancer types in Oncomine and GEPIA were summarized in Supplementary Table 1 and Supplementary Figure S1, respectively. 
Table 1

TSHZ3 expression in different cancer types.

\begin{tabular}{|c|c|c|c|c|c|}
\hline Cancer types & $\mathrm{N}$ (normal) & $\mathrm{N}($ cancer) & Up/Down & Significance & Database \\
\hline ACC & 128 & 77 & Down & \# & GEPIA \\
\hline BLCA & 204 & 339 & Up & * & ONCOMINE \\
\hline Brain and Central Nervous System Cancer & 796 & 1309 & Down & \# & ONCOMINE \\
\hline GBM & 207 & 163 & Up & \# & GEPIA \\
\hline LGG & 207 & 518 & Up & \# & GEPIA \\
\hline Breast Cancer & 1141 & 3628 & Down & \# & ONCOMINE \\
\hline BRCA & 1134 & 3318 & Down & * & ONCOMINE \\
\hline Cervical Cancer & 132 & 245 & Up & * & ONCOMINE \\
\hline CESC & 110 & 225 & Up & \# & ONCOMINE \\
\hline Colorectal Cancer & 728 & 1102 & Down & \# & ONCOMINE \\
\hline COAD & 627 & 542 & Down & \# & ONCOMINE \\
\hline READ & 631 & 278 & Down & \# & ONCOMINE \\
\hline $\mathrm{CHOL}$ & 9 & 36 & Up & \# & GEPIA \\
\hline DLBC & 337 & 47 & Down & \# & GEPIA \\
\hline ESCA & 286 & 182 & Up & \# & GEPIA \\
\hline Gastric Cancer & 573 & 688 & Up & * & ONCOMINE \\
\hline STAD & 478 & 554 & Up & * & ONCOMINE \\
\hline Head and Neck Cancer & 934 & 1068 & Up & \# & ONCOMINE \\
\hline THCA & 551 & 607 & Down & \# & ONCOMINE \\
\hline HNSC & 530 & 516 & Up & \# & ONCOMINE \\
\hline Kidney Cancer & 553 & 598 & Down & \# & ONCOMINE \\
\hline $\mathrm{KICH}$ & 53 & 66 & Down & \# & GEPIA \\
\hline $\mathrm{KIRC}$ & 553 & 502 & Up & \# & ONCOMINE \\
\hline KIRP & 544 & 62 & Down & \# & ONCOMINE \\
\hline Leukemia & 304 & 2073 & Down & * & ONCOMINE \\
\hline LAML & 304 & 757 & Down & \# & ONCOMINE \\
\hline Liver Cancer & 281 & 323 & Up & * & ONCOMINE \\
\hline Lung Cancer & 1018 & 1389 & Up & * & ONCOMINE \\
\hline LUAD & 1018 & 811 & Down & \# & ONCOMINE \\
\hline LUSC & 940 & 551 & Up & * & ONCOMINE \\
\hline Lymphoma & 129 & 247 & Up & \# & ONCOMINE \\
\hline Melanoma & 339 & 95 & Up & * & ONCOMINE \\
\hline Myeloma(multiple myeloma) & 84 & 84 & Up & * & ONCOMINE \\
\hline Endometrial Cancer & 363 & 354 & Up & \# & ONCOMINE \\
\hline
\end{tabular}

*P $<0.05, \# P \geq 0.05, A C C$ (Adrenocortical carcinoma), BLCA(Bladder urothelial carcinoma), BRCA (Breast invasive carcinoma), CESC (Cervical squamous cell carcinoma and endocervical adenocarcinoma), CHOL (Cholangio carcinoma), COAD (Colon adenocarcinoma), DLBC (Lymphoid Neoplasm Diffuse Large B-cell Lymphoma), ESCA (Esophageal carcinoma), GBM (Glioblastoma multiforme), HNSC (Head and Neck squamous cell carcinoma), KICH (Kidney Chromophobe), KIRC (Kidney renal clear cell carcinoma), KIRP (Kidney renal papillary cell carcinoma), LAML (Acute

Myeloid Leukemia), LGG (Brain Lower Grade Glioma), LIHC (Liver hepatocellular carcinoma), LUAD (Lung adenocarcinoma), LUSC (Lung squamous cell carcinoma), MESO (Mesothelioma), OV (Ovarian serous cystadenocarcinoma), PAAD (Pancreatic adenocarcinoma), PCPG

(Pheochromocytoma and Paraganglioma), PRAD (Prostate adenocarcinoma), READ (Rectum adenocarcinoma), SARC (Sarcoma), SKCM (Skin Cutaneous Melanoma), STAD (Stomach adenocarcinoma), TGCT (Testicular Germ Cell Tumors), THCA (Thyroid carcinoma), THYM (Thymoma), UCEC (Uterine Corpus Endometrial Carcinoma), UCS (Uterine Carcinosarcoma). 


\begin{tabular}{|c|c|c|c|c|c|}
\hline Cancer types & N (normal) & $\mathrm{N}$ (cancer) & Up/Down & Significance & Database \\
\hline Skin Cancer & 4 & 26 & Up & * & ONCOMINE \\
\hline SKCM & 558 & 461 & Down & $\#$ & GEPIA \\
\hline TGCT & 165 & 137 & Up & $\#$ & GEPIA \\
\hline THYM & 339 & 118 & Down & $\#$ & GEPIA \\
\hline Ovarian Cancer & 576 & 695 & Down & * & ONCOMINE \\
\hline OV & 561 & 607 & Up & * & ONCOMINE \\
\hline Pancreatic Cancer & 150 & 175 & Up & * & ONCOMINE \\
\hline PAAD & 171 & 179 & Up & * & GEPIA \\
\hline PCPG & 3 & 182 & Up & * & GEPIA \\
\hline Prostate Cancer & 364 & 515 & Down & * & ONCOMINE \\
\hline PRAD & 152 & 492 & Down & * & GEPIA \\
\hline SARC & 2 & 262 & Up & $\#$ & GEPIA \\
\hline UCEC & 91 & 174 & Down & * & GEPIA \\
\hline UCS & 78 & 57 & Down & * & GEPIA \\
\hline \multicolumn{6}{|c|}{$\begin{array}{l}\text { *P }<0.05 \text {, \#P } \geq 0.05 \text {, ACC(Adrenocortical carcinoma), BLCA(Bladder urothelial carcinoma), BRCA (Breast invasive carcinoma), CESC (Cervical } \\
\text { squamous cell carcinoma and endocervical adenocarcinoma), CHOL (Cholangio carcinoma), COAD (Colon adenocarcinoma), DLBC (Lymphoid } \\
\text { Neoplasm Diffuse Large B-cell Lymphoma), ESCA (Esophageal carcinoma), GBM (Glioblastoma multiforme), HNSC (Head and Neck squamous cel } \\
\text { carcinoma), KICH (Kidney Chromophobe), KIRC (Kidney renal clear cell carcinoma), KIRP (Kidney renal papillary cell carcinomama), LAML (Acute } \\
\text { Myeloid Leukemia), LGG (Brain Lower Grade Glioma), LIHC (Liver hepatocellular carcinoma), LUAD (Lung adenocarcinoma), LUSC (Lung squamou } \\
\text { cell carcinoma), MESO (Mesothelioma), OV (Ovarian serous cystadenocarcinoma), PAAD (Pancreatic adenocarcinoma), PCPG } \\
\text { (Pheochromocytoma and Paraganglioma), PRAD (Prostate adenocarcinoma), READ (Rectum adenocarcinoma), SARC (Sarcoma), SKCM (Skin } \\
\text { Cutaneous Melanoma), STAD (Stomach adenocarcinoma), TGCT (Testicular Germ Cell Tumors), THCA (Thyroid carcinoma), THYM (Thymoma), } \\
\text { UCEC (Uterine Corpus Endometrial Carcinoma), UCS (Uterine Carcinosarcoma). }\end{array}$} \\
\hline
\end{tabular}

\section{Prognostic Potential of TSHZ3 in Tumors}

We evaluated the prognostic potential of TSHZ3 in cancers by presenting survival curves via Kaplan-Meier plotter and TIMER. Among the cancers with TSHZ3 differential expressions compared to normal tissues, elevated expression levels of TSHZ3 were correlated with poor prognosis of BLCA (Fig. 2A1), gastric cancer (Fig. 2A2-A3), STAD (Fig. 2A4)and ovarian cancer (Fig. 2B4-C1) but with good prognosis of lung cancer (Fig. 2C1). However, the TSHZ3 expression level has no significance correlation with lung squamous cell carcinoma (LUSC) or ovarian serous cystadenocarcinoma (OV), the main histological types of lung cancer and ovarian cancer, respectively (Fig. 2B3, C2). Notably, high TSHZ3 expression levels were correlated with poor prognosis of OS and PFS in GC (OS HR $=1.96,95 \% \mathrm{Cl}=1.57$ to 2.44, $\mathrm{P}=1.2 \mathrm{e}-9$; $\mathrm{PFS} \mathrm{HR}=2.05,95 \% \mathrm{Cl}=1.61$ to $2.60, \mathrm{P}=2.2 \mathrm{e}-9)$ and ovarian cancer $(\mathrm{OS} \mathrm{HR}=1.42,95 \% \mathrm{Cl}=1.15$ to 1.76, $\mathrm{P}=0.0013$; PFS HR=1.49, 95\% Cl = 1.23 to 1.80, $\mathrm{P}=3.5 \mathrm{e}-5)$ (Fig. 2A2,A3). High TSHZ3 expression also shows poor OS in BLCA (OS HR = 1.96, 95\% Cl=1.008 to 1.217, $P=0.006)$ and $S T A D(O S H R=1.178,95 \% \mathrm{Cl}=1.039$ to $1.336, P=0.009)(F i g .2 A 1, A 4$, Supplementary Table S2).

The relationships between TSHZ3 expression and prognosis of diverse cancers are presented in Fig. 2 and Supplementary Table S2. These results strongly suggest that TSHZ3 is a prognostic factor in many cancer types. The reduced or elevated levels of TSHZ3 may have diverse prognostic value depending on the type of cancer.

\section{High TSHZ3 Expression Influences the Prognosis of Gastric Cancer Patients with Lymphatic Metastasis}

To better understand the association and underlying mechanisms of TSHZ3 expression in GC, we investigated the correlations between the TSHZ3 expression and the related clinical features in the GC patients with the Kaplan-Meier plotter databases. Our results showed that overexpression of TSHZ3 was correlated with worse OS and PFS in female and male patients as well as patients with two types of Lauren classification (diffuse type and intestinal type) $(P<0.05)$. Specifically, in GC patients, high TSHZ3 expression was associated with worse OS and PFS in stage 3 to 4 but was not correlated with OS and PFS of stage 1 (OS HR $=2.16, \mathrm{P}=0.20$; PFS HR $=1.97, \mathrm{P}=0.26$ ) to 2 (OS HR= $1.74, P=0.09 ; \mathrm{PFS} H \mathrm{HR}=1.81, P=0.054)$ and PFS of stage $M 1$ patients (PFS HR = 1.57, $P=0.16$ ) (Table 2). Here the $M$ category refers to distant metastases involvement; $M 0$ indicates no distant metastases and M1 indicate distant metastases [29]. In addition, high TSHZ3 expression level has the highest HR values of OS and PFS in all five N categories. These findings suggest that TSHZ3 expression level is associated with the prognosis of GC patients with lymph node metastases and distant metastases. 
Table 2

Correlation between TSHZ3 expression and prognosis in gastric cancer with different clinicopathological characteristics in Kaplan-Meier plotter

\begin{tabular}{|c|c|c|c|c|c|c|}
\hline \multirow[t]{2}{*}{ Clinicopathological characteristics } & \multicolumn{3}{|c|}{$\begin{array}{l}\text { Overall survival } \\
(n=882)\end{array}$} & \multicolumn{3}{|c|}{$\begin{array}{l}\text { Progression-free survival } \\
(n=646)\end{array}$} \\
\hline & $\mathbf{N}$ & Hazard ratio & $\mathbf{P}$ & $\mathbf{N}$ & Hazard ratio & $\mathbf{P}$ \\
\hline \multicolumn{7}{|l|}{ GENDER } \\
\hline Male & 349 & $2.15(1.60-2.89)$ & $2.1 e-07$ & 341 & $2.15(1.61-2.88)$ & $1.2 e-07$ \\
\hline Female & 187 & $2.52(1.57-4.04)$ & $7.7 e-05$ & 179 & $2.27(1.50-3.46)$ & $7.9 e-05$ \\
\hline \multicolumn{7}{|l|}{ STAGE } \\
\hline 1 & 62 & $2.16(0.65-7.22)$ & 0.20 & 60 & $1.97(0.59-6.59)$ & 0.26 \\
\hline 2 & 135 & $1.74(0.91-3.31)$ & 0.09 & 131 & $1.81(0.98-3.34)$ & 0.054 \\
\hline 3 & 197 & $1.99(1.36-2.91)$ & 0.00031 & 186 & $2.24(1.52-3.3)$ & $2.8 e-05$ \\
\hline 4 & 140 & $1.98(1.31-3.01)$ & 0.0011 & 141 & $1.51(1.03-2.23)$ & 0.034 \\
\hline \multicolumn{7}{|l|}{ STAGE T } \\
\hline 2 & 241 & $2.08(1.36-3.2)$ & 0.00062 & 239 & $1.98(1.31-3.00)$ & 0.001 \\
\hline 3 & 204 & $1.70(1.17-2.47)$ & 0.0047 & 204 & $1.49(1.04-2.12)$ & 0.027 \\
\hline 4 & 38 & $3.06(1.25-7.46)$ & 0.0099 & 39 & $4.39(1.75-11.02)$ & 0.00074 \\
\hline \multicolumn{7}{|l|}{ STAGE N } \\
\hline 0 & 74 & $2.66(1.1-6.4)$ & 0.025 & 72 & $2.62(1.09-6.29)$ & 0.025 \\
\hline 1 & 225 & $2.53(1.68-3.82)$ & $4.6 e-06$ & 222 & $2.38(1.61-3.52)$ & $7.8 e-06$ \\
\hline 2 & 121 & $2.14(1.36-3.37)$ & 0.00077 & 125 & $2.06(1.32-3.23)$ & 0.0012 \\
\hline 3 & 76 & $2.34(1.35-4.06)$ & 0.0019 & 76 & $2(1.16-3.45)$ & 0.012 \\
\hline $1+2+3$ & 442 & $2.29(1.75-2.98)$ & $3.6 e-10$ & 423 & $2.12(1.65-2.74)$ & $2.9 e-09$ \\
\hline \multicolumn{7}{|l|}{ STAGE M } \\
\hline 0 & 444 & $2.2(1.67-2.92)$ & $1.5 e-08$ & 443 & $2.07(1.59-2.71)$ & $5.3 e-08$ \\
\hline 1 & 56 & $2.46(1.33-4.56)$ & 0.0033 & 56 & $1.57(0.84-2.93)$ & 0.16 \\
\hline \multicolumn{7}{|l|}{ LAUREN CLASSIFICATION } \\
\hline Diffuse & 240 & $1.95(1.36-2.8)$ & 0.00022 & 231 & $2.4(1.64-3.52)$ & $3.4 e-06$ \\
\hline Intestinal & 169 & $2.18(1.51-3.13)$ & $1.8 e-05$ & 263 & $2.17(1.52-3.08)$ & $1.1 e-05$ \\
\hline Mixed & 29 & $3.84(1.24-11.9)$ & 0.013 & 28 & $1.98(0.56-7)$ & 0.28 \\
\hline \multicolumn{7}{|l|}{ DIFFERENTIATION } \\
\hline Poor & 121 & $1.57(0.96-2.59)$ & 0.071 & 121 & $1.52(0.95-2.43)$ & 0.08 \\
\hline Moderate & 67 & $2.17(0.94-5.02)$ & 0.064 & 67 & $1.83(0.98-3.41)$ & 0.055 \\
\hline
\end{tabular}

\section{TSHZ3 Expression is Associated with Immune Infiltrates Level in STAD and BLCA}

We evaluated the correlations between TSHZ3 expression and immune infiltration profiles in 39 cancer types with TIMER. The rough evaluation results revealed significant correlations between TSHZ3 expression and the tumor purity in 25 cancer types, B cell infiltration levels in 10 cancer types, CD $8+T$ cells infiltrating levels in 18 cancer types, CD4 + T cells infiltrating levels in 24 cancer types, macrophages infiltrating levels in 25 cancer types, neutrophils infiltrating levels in 26 cancer types, and dendritic cells infiltrating levels in 27 cancer types (Fig. 3 and Supplementary Fig. 2).

Based on the correlation between TSHZ3 expression and immune infiltration profiles in different cancer types, we next focused on the distinct cancer types in which TSHZ3 was correlated with prognosis and immune infiltrates. Tumor purity can affect the results of immune infiltration analysis in clinical cancer samples with genomic approaches [30]. Thus, we selected the cancer types with significant negative correlation between TSHZ3 expression and tumor purity in TIMER to further exploration. Interestingly, we found there were significant positive correlations between TSHZ3 
expression levels and infiltrating levels of CD8 $+\mathrm{T}$ cells $(r=0.235, P=4.89 \mathrm{e}-06), \mathrm{CD} 4+\mathrm{T}$ cells $(r=0.455, P=3.92 \mathrm{e}-20)$, macrophages $(r=0.696, P=$ 5.68e- 55), neutrophils ( $r=0.332, P=5.54 \mathrm{e}-11)$ and DCs $(r=0.476, P=2.28 \mathrm{e}-22)$ in STAD (Fig. 3-C). Similarly, significant positive correlations existed between TSHZ3 expression and infiltrating levels of CD4 + T cells $(r=0.186, P=3.62 \mathrm{e}-04)$, macrophages $(r=0.360, P=1.25 \mathrm{e}-12)$, neutrophils $(r=0.163$, $P=1.79 \mathrm{e}-03)$, and DCs $(r=0.130, P=1.26 \mathrm{e}-02)$ in BLCA (Fig. 3-A). Furthermore, there was no significant associations between TSHZ3 expression and infiltrating levels of CD8 + T cells, B cells, CD4 + T cells, macrophages and DCs in kidney chromophobe (KICH) (Fig. 3-B).

\section{Refined Analysis on the Correlation between TSHZ3 Expression and Immune Infiltrates}

To further investigate the correlations between TSHZ3 expression and immune infiltrates, we focused on the correlations between TSHZ3 and immune marker genes of diverse TIICs and its' subtypes of BLCA, STAD in TIMER using KICH as the control. These TIICs included TAMs, B cells, monocytes, neutrophils, NK cells, CD8 + T cells and T cells (general). As different subsets of some TIICs have diverse effects in the progression of cancers, we also analyzed subsets of different TIICs, including M1 and M2 macrophages, pDCs and cDC1s, Th1 cells, Th2 cells, Th17 cells, Tregs, Tfh cells as well as T cells exhaustion. After adjusted with purity, our results revealed significant correlations between TSHZ3 expression levels and all listed immune marker sets in some pro-tumorigenic TIICs in STAD and BLCA, such as M2 macrophages, monocytes, B cells and pDCs. Besides, the expressions of all the immune marker sets of other TIICs (TAMs, Th2 cells and Tregs) were positively correlated with theTSHZ3 expression level in STAD. However, TSHZ3 expression level had no significant correlation with TIICs in $\mathrm{KICH}$ (Table 3). The validation results via GEPIA were similar to the refined analysis results in TIMER (Table 4). 
Table 3

Correlation analysis between TSHZ3 and gene markers of immune cells in TIMER

\begin{tabular}{|c|c|c|c|c|c|c|c|c|c|c|c|c|c|}
\hline \multirow{3}{*}{$\begin{array}{l}\text { Immune } \\
\text { Cells }\end{array}$} & \multirow[t]{3}{*}{ Gene Markers } & \multicolumn{4}{|l|}{ STAD } & \multicolumn{4}{|l|}{ BLCA } & \multicolumn{4}{|l|}{$\mathrm{KICH}$} \\
\hline & & \multicolumn{2}{|l|}{ None } & \multicolumn{2}{|l|}{ Purity } & \multicolumn{2}{|l|}{ None } & \multicolumn{2}{|l|}{ Purity } & \multicolumn{2}{|l|}{ None } & \multicolumn{2}{|l|}{ Purity } \\
\hline & & Cor & $\mathbf{P}$ & Cor & $\mathbf{P}$ & Cor & $\mathbf{P}$ & Cor & $\mathbf{P}$ & Cor & $\mathbf{P}$ & Cor & $\mathbf{P}$ \\
\hline \multirow[t]{4}{*}{ TAM } & CCL2 & 0.539 & $\star \star \star *$ & 0.511 & $\star \star \star *$ & 0.500 & $\star \star \star *$ & 0.327 & 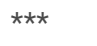 & -0.078 & 0.531 & -0.076 & 0.546 \\
\hline & CD68 & 0.231 & $\star \star \star$ & 0.203 & $\star \star \star ~$ & 0.284 & $\star \star \star$ & 0.097 & 0.063 & 0.001 & 0.995 & 0.017 & 0.893 \\
\hline & IL6 & 0.297 & $\star \star \star *$ & 0.271 & $\star \star \star \star$ & 0.426 & $\star \star \star$ & 0.242 & 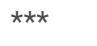 & 0.128 & 0.307 & 0.144 & 0.252 \\
\hline & IL10 & 0.457 & $\star \star \star *$ & 0.440 & $\star * *$ & 0.562 & $\star \star \star *$ & 0.413 & $\star \star \star *$ & 0.044 & 0.726 & 0.068 & 0.593 \\
\hline \multirow[t]{3}{*}{ B Cell } & CD19 & 0.332 & $\star \star \star$ & 0.307 & $\star \star \star$ & 0.332 & $\star \star \star ~$ & 0.141 & $\star \star$ & 0.067 & 0.594 & 0.076 & 0.549 \\
\hline & CD79A & 0.345 & $\star \star \star ~$ & 0.302 & $\star \star \star *$ & 0.398 & 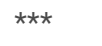 & 0.203 & $\star \star \star ~$ & 0.165 & 0.186 & 0.179 & 0.154 \\
\hline & PNOC & 0.383 & $\star \star \star \star ~$ & 0.349 & $\star \star \star *$ & 0.380 & 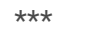 & 0.217 & 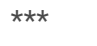 & 0.092 & 0.462 & 0.102 & 0.419 \\
\hline \multirow[t]{5}{*}{$\mathrm{pDC}$} & CD303(CLEC4C) & 0.336 & $\star \star \star$ & 0.355 & $\star \star \star$ & 0.275 & $\star \star \star$ & 0.153 & ** & 0.119 & 0.341 & 0.126 & 0.319 \\
\hline & BDCA-4(NRP1) & 0.674 & $\star \star \star *$ & 0.660 & $\star \star \star *$ & 0.445 & $\star \star \star *$ & 0.334 & 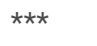 & 0.162 & 0.192 & 0.201 & 0.108 \\
\hline & CD304(IL3RA) & 0.632 & $\star \star \star$ & 0.609 & $\star \star \star$ & 0.483 & $\star \star \star *$ & 0.318 & $\star \star \star ~$ & 0.036 & 0.776 & 0.040 & 0.755 \\
\hline & CCR2 & 0.475 & $\star \star \star \star ~$ & 0.451 & $\star \star \star \star ~$ & 0.463 & $\star \star \star ~$ & 0.225 & 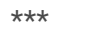 & 0.030 & 0.809 & 0.053 & 0.676 \\
\hline & CXCR3 & 0.255 & $\star \star *$ & 0.230 & $\star \star \star$ & 0.362 & $\star \star \star$ & 0.128 & * & 0.011 & 0.933 & 0.025 & 0.842 \\
\hline \multirow[t]{5}{*}{$\mathrm{CDC1}$} & HLA-DRA & 0.205 & $\star \star \star *$ & 0.168 & ** & 0.333 & $\star \star \star ~$ & 0.098 & 0.059 & -0.147 & 0.238 & -0.164 & 0.192 \\
\hline & CD141(THBD) & 0.613 & $\star \star \star ~$ & 0.599 & $\star \star \star *$ & 0.121 & * & 0.029 & 0.578 & 0.061 & 0.625 & 0.075 & 0.554 \\
\hline & XCR1 & 0.342 & $\star \star * *$ & 0.317 & $\star * *$ & 0.218 & $\star \star \star *$ & 0.164 & ** & 0.311 & * & 0.311 & * \\
\hline & CLEC9A & 0.363 & $\star \star \star$ & 0.347 & $\star \star \star$ & 0.336 & $\star \star \star$ & 0.182 & $\star \star \star$ & -0.330 & $\star \star$ & -0.346 & $\star \star$ \\
\hline & DEC-205(LY75) & -0.132 & ** & -0.138 & ** & -0.045 & 0.368 & -0.032 & 0.544 & -0.083 & 0.508 & -0.081 & 0.522 \\
\hline \multirow[t]{3}{*}{ Monocyte } & CD155(CSF1R) & 0.537 & $* * *$ & 0.520 & $\star \star * *$ & 0.553 & $\star \star \star *$ & 0.385 & $\star \star * *$ & -0.171 & 0.170 & -0.199 & 0.112 \\
\hline & CD86 & 0.409 & $\star \star \star$ & 0.382 & $\star \star \star$ & 0.478 & $\star \star \star$ & 0.275 & $\star \star \star$ & -0.080 & 0.532 & -0.081 & 0.519 \\
\hline & CCR1 & 0.415 & $\star \star \star *$ & 0.388 & $\star \star *$ & 0.454 & $\star \star \star$ & 0.258 & $\star \star \star$ & -0.095 & 0.446 & -0.097 & 0.442 \\
\hline \multirow{3}{*}{$\begin{array}{l}\text { M1 } \\
\text { Macrophage }\end{array}$} & IRF5 & 0.219 & $\star \star \star ~$ & 0.196 & $\star \star \star ~$ & -0.030 & 0.545 & -0.025 & 0.628 & -0.221 & 0.075 & -0.238 & 0.056 \\
\hline & INOS(NOS2) & -0.012 & 0.813 & -0.025 & 0.628 & 0.123 & * & 0.049 & 0.350 & -0.186 & 0.134 & -0.192 & 0.125 \\
\hline & COX2(PTGS2) & 0.270 & $\star \star \star$ & 0.254 & $\star \star \star$ & 0.178 & $\star \star \star$ & 0.115 & * & 0.068 & 0.584 & 0.068 & 0.592 \\
\hline \multirow{5}{*}{$\begin{array}{l}\text { M2 } \\
\text { Macrophage }\end{array}$} & IRF4 & 0.327 & $\star \star \star$ & 0.285 & $\star \star \star$ & 0.411 & $\star \star \star$ & 0.183 & 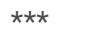 & -0.021 & 0.866 & -0.011 & 0.931 \\
\hline & CCL17 & 0.271 & $\star \star \star *$ & 0.231 & $\star * \star$ & 0.204 & $\star \star \star *$ & 0.134 & * & 0.039 & 0.754 & 0.048 & 0.703 \\
\hline & CD163 & 0.469 & $\star \star \star *$ & 0.455 & $\star \star \star *$ & 0.535 & $\star \star \star *$ & 0.352 & $\star * \star$ & 0.003 & 0.982 & 0.018 & 0.888 \\
\hline & VSIG4 & 0.495 & $\star \star \star *$ & 0.497 & $\star \star \star *$ & 0.530 & $\star \star \star *$ & 0.351 & $\star \star \star \star ~$ & -0.019 & 0.877 & -0.010 & 0.936 \\
\hline & MS4A4A & 0.515 & $\star \star \star$ & 0.504 & $\star \star \star$ & 0.539 & $\star \star \star$ & 0.360 & $\star \star \star$ & -0.072 & 0.565 & -0.074 & 0.560 \\
\hline \multirow{7}{*}{$\begin{array}{l}\text { Natural } \\
\text { Killer Cell }\end{array}$} & KIR2DL1 & 0.140 & $\star \star$ & 0.130 & * & 0.125 & * & -0.022 & 0.680 & -0.085 & 0.499 & -0.082 & 0.519 \\
\hline & KIR2DL3 & 0.061 & 0.213 & 0.023 & 0.658 & 0.174 & $* \star *$ & -0.001 & 0.991 & -0.211 & 0.089 & -0.210 & 0.926 \\
\hline & KIR2DL4 & -0.074 & 0.131 & -0.114 & * & 0.118 & * & -0.049 & 0.351 & -0.185 & 0.137 & -0.184 & 0.143 \\
\hline & KIR3DL1 & 0.100 & * & 0.080 & 0.119 & 0.168 & 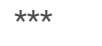 & 0.077 & 0.141 & -0.120 & 0.338 & -0.118 & 0.350 \\
\hline & KIR3DL2 & 0.139 & $\star \star$ & 0.110 & * & 0.095 & 0.056 & -0.057 & 0.273 & -0.051 & 0.682 & -0.047 & 0.710 \\
\hline & KIR3DL3 & -0.078 & 0.112 & -0.069 & 0.177 & -0.038 & 0.445 & -0.112 & * & -0.250 & * & -0.250 & * \\
\hline & KIR2DS4 & 0.034 & 0.487 & 0.014 & 0.791 & 0.103 & * & -0.053 & 0.309 & -0.131 & 0.295 & -0.130 & 0.302 \\
\hline
\end{tabular}

${ }^{\star} \mathrm{P}<0.05,{ }^{\star \star} \mathrm{P}<0.01,{ }^{\star \star *} \mathrm{P}<0.001 ; \mathrm{NA}$ or NaN means no association (the expression level of marker was 0); STAD, stomach adenocarcinoma; BLCA, bladder urothelial carcinoma; $\mathrm{KICH}$, kidney chromophobe; TAM, tumor-associated macrophage; pDC, plasmacytoid dendritic cell; Th, T helper cell; Tfh, Follicular helper T cell; Treg, regulatory T cell; None, correlation without adjustment. Purity, correlation adjusted by purity; Cor, R value of Spearman's correlation. 


\begin{tabular}{|c|c|c|c|c|c|c|c|c|c|c|c|c|c|}
\hline \multirow[t]{3}{*}{ Neutrophils } & CCR7 & 0.428 & 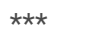 & 0.389 & $\star \star \star *$ & 0.139 & $\star *$ & 0.078 & 0.133 & 0.100 & 0.424 & 0.125 & 0.320 \\
\hline & CD66b(CEACAM8) & 0.007 & 0.890 & 0.018 & 0.727 & 0.017 & 0.739 & 0.023 & 0.654 & -0.011 & 0.928 & -0.011 & 0.929 \\
\hline & CD11b(ITGAM) & 0.513 & $\star \star \star ~$ & 0.502 & $\star \star \star ~$ & 0.514 & $\star \star \star$ & 0.328 & $\star \star \star \star$ & -0.135 & 0.278 & -0.158 & 0.208 \\
\hline \multirow{2}{*}{$\begin{array}{l}\text { CD8 + T } \\
\text { Cell }\end{array}$} & CD8A & 0.260 & $\star \star \star$ & 0.228 & 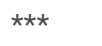 & 0.260 & $* * \star$ & 0.028 & 0.592 & 0.058 & 0.631 & 0.092 & 0.467 \\
\hline & CD8B & 0.108 & * & 0.081 & 0.114 & 0.148 & $\star *$ & -0.025 & 0.638 & 0.233 & 0.060 & 0.290 & * \\
\hline \multirow{3}{*}{$\begin{array}{l}\text { T Cell } \\
\text { (general) }\end{array}$} & $\mathrm{CD} 2$ & 0.270 & $\star \star \star$ & 0.231 & 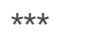 & 0.349 & $* \star \star$ & 0.105 & * & 0.069 & 0.578 & 0.100 & 0.427 \\
\hline & CD3D & 0.225 & $\star \star \star$ & 0.174 & 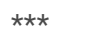 & 0.314 & $\star \star \star$ & 0.098 & 0.061 & 0.040 & 0.747 & 0.063 & 0.617 \\
\hline & CD3E & 0.253 & $\star \star \star$ & 0.206 & $\star \star \star ~$ & 0.371 & $\star \star \star$ & 0.125 & * & 0.028 & 0.821 & 0.048 & 0.707 \\
\hline \multirow[t]{5}{*}{ Th1 } & STAT1 & -0.061 & 0.214 & -0.067 & 0.192 & 0.158 & $\star \star$ & -0.046 & 0.378 & -0.119 & 0.341 & -0.117 & 0.354 \\
\hline & STAT4 & 0.329 & $\star \star \star$ & 0.300 & $\star \star \star ~$ & 0.352 & 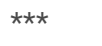 & 0.118 & * & -0.070 & 0.575 & -0.069 & 0.584 \\
\hline & T-bet(TBX21) & 0.268 & $\star \star \star *$ & 0.235 & $\star \star \star *$ & 0.315 & $\star \star \star *$ & 0.087 & 0.097 & -0.205 & 0.099 & -0.221 & 0.077 \\
\hline & IFN- $\gamma($ IFNG) & -0.067 & 0.17 & -0.096 & 0.061 & 0.135 & ** & -0.069 & 0.187 & 0.159 & 0.203 & 0.195 & 0.120 \\
\hline & TNF-a(TNF) & 0.128 & ** & 0.085 & 0.099 & 0.154 & $\star \star$ & 0.006 & 0.907 & -0.206 & 0.097 & -0.205 & 0.085 \\
\hline \multirow[t]{4}{*}{ Th2 } & IL13 & 0.142 & $\star \star$ & 0.146 & ** & 0.169 & $* \star *$ & 0.037 & 0.485 & -0.039 & 0.755 & -0.040 & 0.750 \\
\hline & STAT5A & 0.409 & $\star \star \star$ & 0.403 & $\star \star \star$ & 0.173 & $\star \star \star$ & -0.001 & 0.990 & -0.201 & 0.106 & -0.216 & 0.084 \\
\hline & STAT6 & 0.122 & * & 0.120 & * & -0.089 & 0.074 & -0.003 & 0.957 & -0.194 & 0.118 & -0.201 & 0.108 \\
\hline & GATA3 & 0.361 & $\star \star \star$ & 0.346 & $\star \star \star \star$ & -0.215 & $\star \star \star \star$ & -0.08 & 0.126 & -0.374 & $\star \star$ & -0.374 & *夫 \\
\hline \multirow[t]{2}{*}{ Th17 } & IL17A & -0.197 & $\star \star \star *$ & -0.227 & $\star \star \star *$ & -0.065 & 0.190 & -0.127 & * & NA & NA & $\mathrm{NaN}$ & $\mathrm{NaN}$ \\
\hline & STAT3 & 0.454 & $\star \star \star ~$ & 0.437 & $\star \star \star ~$ & 0.231 & $\star \star \star$ & 0.072 & 0.166 & -0.135 & 0.277 & -0.140 & 0.267 \\
\hline \multirow[t]{2}{*}{ Tfh } & IL21 & 0.076 & 0.124 & 0.057 & 0.273 & 0.114 & * & 0.035 & 0.505 & NA & NA & $\mathrm{NaN}$ & $\mathrm{NaN}$ \\
\hline & BCL6 & 0.534 & 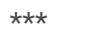 & 0.512 & $\star \star \star *$ & -0.046 & 0.353 & -0.009 & 0.856 & 0.137 & 0.272 & 0.135 & 0.283 \\
\hline \multirow[t]{4}{*}{ Treg } & CCR8 & 0.358 & $\star \star \star \star ~$ & 0.343 & $\star \star \star *$ & 0.426 & $\star \star \star *$ & 0.282 & $\star \star \star \star$ & 0.167 & 0.179 & 0.179 & 0.154 \\
\hline & FOXP3 & 0.242 & 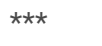 & 0.199 & $\star \star \star *$ & 0.393 & 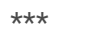 & 0.220 & $\star \star \star ~$ & 0.212 & 0.087 & 0.230 & 0.065 \\
\hline & STAT5B & 0.608 & $\star \star \star \star ~$ & 0.591 & $\star \star \star ~$ & 0.098 & * & 0.077 & 0.142 & -0.193 & 0.121 & -0.192 & 0.125 \\
\hline & TGF $\beta$ (TGFB1) & 0.641 & $\star \star \star \star ~$ & 0.633 & $\star \star \star *$ & 0.308 & 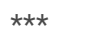 & 0.226 & $\star \star \star ~$ & 0.528 & $\star \star \star \star ~$ & 0.555 & $\star \star \star \star$ \\
\hline \multirow{5}{*}{$\begin{array}{l}\text { T Cell } \\
\text { Exhaustion }\end{array}$} & CTLA4 & 0.104 & * & 0.061 & 0.235 & 0.300 & $\star \star \star ~$ & 0.071 & 0.177 & 0.053 & 0.672 & 0.073 & 0.565 \\
\hline & GZMB & 0.011 & 0.817 & 0.044 & 0.388 & 0.286 & 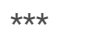 & 0.035 & 0.504 & -0.060 & 0.631 & -0.056 & 0.659 \\
\hline & LAG3 & 0.112 & * & 0.076 & 0.137 & 0.259 & 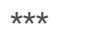 & 0.037 & 0.438 & 0.075 & 0.548 & 0.086 & 0.498 \\
\hline & PD-1(PDCD1) & 0.174 & $\star \star \star$ & 0.139 & $\star \star$ & 0.311 & $\star \star \star$ & 0.081 & 0.123 & 0.057 & 0.651 & 0.075 & 0551 \\
\hline & TIM-3(HAVCR2) & 0.392 & $\star \star \star$ & 0.379 & $\star \star \star ~$ & 0.458 & $\star \star \star$ & 0.239 & $\star \star *$ & -0.127 & 0.309 & -0.131 & 0.299 \\
\hline
\end{tabular}


Table 4

Correlation analysis between TSHZ3 and relate gene markers of immune cell in GEPIA.

\begin{tabular}{|c|c|c|c|c|c|c|c|c|c|c|c|c|c|}
\hline \multirow[t]{3}{*}{ Immune Cells } & \multirow[t]{3}{*}{ Gene Markers } & \multicolumn{4}{|l|}{ STAD } & \multicolumn{4}{|l|}{ BLCA } & \multicolumn{4}{|l|}{$\mathrm{KICH}$} \\
\hline & & \multicolumn{2}{|l|}{ Tumor } & \multicolumn{2}{|l|}{ Normal } & \multicolumn{2}{|l|}{ Tumor } & \multicolumn{2}{|l|}{ Normal } & \multicolumn{2}{|l|}{ Tumor } & \multicolumn{2}{|l|}{ Normal } \\
\hline & & $\mathbf{R}$ & $\mathbf{P}$ & $\mathbf{R}$ & $\mathbf{P}$ & $\mathbf{R}$ & $\mathbf{P}$ & $\mathbf{R}$ & $\mathbf{P}$ & $\mathbf{R}$ & $\mathbf{P}$ & $\mathbf{R}$ & $\mathbf{P}$ \\
\hline \multirow[t]{4}{*}{ TAM } & CCL2 & 0.550 & $\star \star \star ~$ & 0.560 & $\star \star \star$ & & & & & -0.024 & 0.980 & 0.440 & * \\
\hline & CD68 & 0.300 & $\star \star \star ~$ & -0.480 & $\star \star$ & & & & & 0.100 & 0.420 & 0.510 & * \\
\hline & IL6 & 0.330 & $\star \star \star$ & 0.750 & $\star \star \star *$ & & & & & 0.140 & 0.260 & 0.240 & 0.250 \\
\hline & IL10 & 0.510 & $\star \star \star$ & -0.021 & 0.90 & & & & & 0.120 & 0.340 & 0.440 & * \\
\hline \multirow[t]{3}{*}{ B Cell } & CD19 & 0.330 & $\star * \star$ & -0.480 & $\star \star$ & 0.360 & 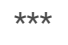 & -0.092 & 0.710 & 0.150 & 0.240 & 0.570 & ** \\
\hline & CD79A & 0.310 & $\star \star \star$ & -0.640 & $\star \star \star$ & 0.410 & $\star \star \star$ & -0.360 & 0.130 & 0.280 & 0.024 & 0.470 & * \\
\hline & PNOC & 0.380 & $\star * \star$ & -0.220 & 0.210 & 0.390 & $\star \star \star$ & 0.340 & $\star \star \star$ & 0.190 & 0.140 & 0.130 & 0.540 \\
\hline \multirow[t]{5}{*}{ pDC } & CD303(CLEC4C) & 0.370 & $\star \star \star$ & 0.098 & 0.570 & 0.340 & $\star \star \star$ & -0.150 & 0.540 & 0.200 & 0.110 & 0.530 & $\star \star \star$ \\
\hline & BDCA-4(NRP1) & 0.710 & $\star \star \star$ & 0.800 & $\star \star \star *$ & 0.470 & $\star \star \star$ & 0.730 & $\star \star \star$ & 0.300 & * & 0.710 & $\star \star \star \star$ \\
\hline & CD304(IL3RA) & 0.680 & $\star \star \star$ & 0.720 & $\star \star \star ~$ & 0.500 & $\star \star \star$ & 0.350 & 0.150 & 0.170 & 0.170 & 0.160 & 0.430 \\
\hline & CCR2 & 0.520 & $\star \star \star ~$ & -0.350 & * & 0.490 & $\star \star \star$ & -0.22 & 0.360 & 0.150 & 0.240 & 0.640 & $\star \star \star$ \\
\hline & CXCR3 & 0.280 & $\star \star \star$ & -0.570 & $\star \star \star$ & 0.400 & $\star \star \star$ & -0.700 & $\star \star \star$ & 0.120 & 0.330 & 0.540 & $\star \star \star \star ~$ \\
\hline \multirow[t]{3}{*}{ Monocyte } & CD155(CSF1R) & 0.600 & $\star \star \star$ & 0.100 & 0.550 & 0.590 & $\star \star \star$ & 0.580 & ** & -0.038 & 0.760 & 0.740 & $* * *$ \\
\hline & CD86 & 0.440 & $\star \star \star ~$ & -0.290 & 0.091 & 0.520 & $\star \star \star$ & -0.320 & 0.180 & 0.023 & 0.860 & 0.510 & ** \\
\hline & CCR1 & 0.480 & $\star \star \star$ & 0.160 & 0.360 & 0.500 & $\star \star \star$ & 0.370 & $\star \star \star$ & 0.025 & 0.840 & 0.370 & 0.068 \\
\hline \multirow[t]{5}{*}{ M2 Macrophage } & IRF4 & 0.350 & $\star \star \star *$ & -0.610 & 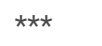 & 0.430 & $\star \star \star$ & -0.380 & 0.100 & 0.063 & 0.620 & 0.730 & $\star \star \star$ \\
\hline & CCL17 & 0.290 & $\star \star \star$ & -0.210 & 0.210 & 0.210 & $\star \star \star$ & 0.190 & * & 0.018 & 0.890 & 0.310 & 0.130 \\
\hline & CD163 & 0.490 & $\star \star \star$ & 0.620 & $\star \star \star \star ~$ & 0.550 & $\star \star \star$ & 0.550 & * & 0.095 & 0.450 & 0.690 & $\star \star \star$ \\
\hline & VSIG4 & 0.530 & $\star \star \star ~$ & 0.350 & * & 0.560 & $\star \star \star ~$ & 0.720 & $\star \star \star \star ~$ & 0.110 & 0.380 & 0.580 & ** \\
\hline & MS4A4A & 0.550 & $\star \star \star$ & 0.470 & $\star \star$ & 0.570 & $\star \star \star$ & 0.750 & $\star \star \star$ & 0.071 & 0.570 & 0.520 & $\star *$ \\
\hline \multirow[t]{4}{*}{ Th2 } & IL13 & 0.230 & $\star \star \star$ & 0.410 & * & & & & & 0.130 & 0.290 & 0.290 & 0.150 \\
\hline & STAT5A & 0.500 & $\star \star \star$ & 0.830 & $\star \star \star$ & & & & & 0.038 & 0.760 & 0.740 & $\star \star \star$ \\
\hline & STAT6 & 0.210 & $\star \star \star$ & 0.610 & $\star * *$ & & & & & 0.061 & 0.630 & 0.810 & $\star \star *$ \\
\hline & GATA3 & 0.360 & $\star \star \star$ & -0.260 & 0.130 & & & & & -0.300 & * & -0.059 & 0.780 \\
\hline \multirow[t]{4}{*}{ Treg } & CCR8 & 0.430 & $\star * \star$ & -0.250 & 0.140 & & & & & 0.230 & 0.059 & 0.110 & 0.580 \\
\hline & FOXP3 & 0.300 & $\star \star \star$ & -0.490 & $\star \star$ & & & & & 0.240 & 0052 & 0.520 & $\star *$ \\
\hline & STAT5B & 0.660 & $\star * \star$ & 0.930 & $\star \star *$ & & & & & 0.092 & 0.460 & 0.770 & $\star * \star$ \\
\hline & TGF $\beta$ (TGFB1) & 0.670 & $\star * *$ & 0.540 & $\star \star \star \star$ & & & & & 0.580 & $\star \star \star *$ & 0.750 & $\star \star \star$ \\
\hline
\end{tabular}

\subsection{Macrophages Infiltrations}

Rough evaluation by the Gene module suggested high TSHZ3 expression was correlated with high infiltration level of macrophages. Refined analyses by the Correlation module revealed high TSHZ3 expression was associated with high infiltration level of M2 macrophages but had no correlations with the infiltration level of M1 Macrophages in STAD and BLCA (Fig. 4A, Table 2). Positive correlation between TAMs infiltration level and TSHZ3 expression in STAD was also revealed (Fig. 4B). These findings imply that TSHZ3 palys a critical role in regulating macrophage polarization in STAD and BLCA.

\subsection{DCs Infiltrations}

Rough evaluation in TIMER showed a high TSHZ3 expression was associated with high infiltration level of DCs. Further refined analyses revealed high TSHZ3 expression was correlated to high infiltration level of pDCs but had no associations with the infiltration level of cDCs1 in STAD and BLCA 
(Fig. 4C, Table 2). These results further suggest that there is a correlation between TSHZ3 and pDCs infiltration in STAD and BLCA.

\subsection{TILs Infiltrations}

Refined analyses revealed high infiltration level of Th2 cells and Tregs correlate with high TSHZ3 expression in STAD (Fig. 4D, Fig. 4E and Table 2), those two subsets are well documented to be pro-tumorigenic. As for the anti-tumorigenic TILs, such as Th1 cells and Tfh cells, their infiltration levels had no significant correlation with TSHZ3 expression in STAD and BLCA (Table 2). In addition, PD-1(PDCD1) and TIM-3(HAVCR2, the crucial genes that regulate T cell exhaustion, had correlation with TSHZ3 expression in STAD (Table 2). Therefore, these findings further confirm that TSHZ3 plays an important role in immune escape in the GC microenvironment.

\subsection{Other TIICs infiltrations}

Other TIICs with pro-tumorigenic effect, including monocytes and B cells, have high infiltration level in STAD and BLCA which present high TSHZ3 expression (Fig. 4F, Fig. 4G). Our results also suggest that infiltrations of other TIICs, such as NK cells, Th 17 cells, Tfh cells and neutrophils has no association with TSHZ3 expression level (Table 2).

\section{Discussion}

TSHZ3 is a driver of the $19 q 12$ amplicon and the silencing of TSHZ3 is selectively lethal in breast cancer cells harbouring its amplification [30]. Additionally, high expression level of TSHZ3 predicts poor prognosis in tongue squamous cell carcinomas by slight correlation with immune infiltration [20]. Here, we report that TSHZ3 expression is up-regulated in many cancer types, such as STAD and BLCA. Furthermore, high expression of TSHZ3 predicts poor prognosis in those two cancers. Our findings are in consistence with the previous studies [20]. Since the TSHZ3 expression levels in different cancer types remain controversial, we also conducted meta-analysis with Oncomine to provide more convinced evidence on the expression levels of TSHZ3 in different cancer types.

The survival analysis in relation to the TSHZ3 expression differs in different databases, we chose the databases with a larger sample size to conduct the survival analyses and we also selected the cancer types whose prognosis have significant correlation with TSHZ3 expression both revealed by the two methods in TIMER to further exploration. Our study demonstrated that TSHZ3 is a potential biomarker to predict poor prognosis in STAD and BLCA. Interestingly, increased TSHZ3 expression levels is able to influence the prognosis of GC patients with lymph node metastasis or distant metastasis, indicating that the TSHZ3 expression can be also used as a predictor for cancer metastasis. Immune infiltration analysis by the Gene module roughly presents the immune infiltrates. Gene makers listed is the sufficient evidence of relevant TIICs infiltration, furthermore, different subsets of TIICs have different effects on the progression of cancers. Thus, we eventually identified immune infiltrates by immune infiltration analysis with the Correlation module of TIMER. In our present study, we found M2 macrophages infiltration, instead of M1 macrophages, is directly correlated with TSHZ3 expression in STAD and BLCA. It is well-documented that M2 macrophages function as pro-tumorigenic by producing Th2 cytokines. In contrast, M1 macrophages are anti-tumorigenic as they generate Th1 cytokines and express tumoricidal phenotype molecules, such as iNOS [13]. In addition, our data show that TAMs infiltration has positive correlation with TSHZ3 expression in STAD. Recent studies report that TAMs is similar to M2 macrophages in phenotypic characteristics and they promote angiogenesis and invasion in tumors [31, 32]. Our data also show that monocytes infiltrations are positively correlated with TSHZ3 expressions in STAD and BLCA. It has been reported that monocytes present pro-tumorigenic effect by promoting tumorigenesis and angiogenesis, inhibiting the antitumor immune response in vivo, and differentiate into TAMs [33, 34].

TILs play key roles in the regulation of tumor microenvironment. As subsets of CD4 + T cells, Th1 cells are anti-tumorigenic, Th2 cells are protumorigenic, and Treg cells usually exert pro-tumorigenic effects by suppressing immune-surveillance [13]. Our results reveal that the infiltrations of Th2 cells and Treg cells are directly correlated with TSHZ3 expression in STAD but the infiltrations of Th1 cells are not. We suggest that high expression of TSHZ3 leads to poor prognosis by promoting Th2 cells and Treg cells infiltrations. B cells promote cancer growth by producing protumorigenic cytokines and altering ratios of Th1 cells: Th2 cells. B cells support tumor growth in B cell-deficient mice by present resistance to engraftment of syngeneic cancers [13,35]. Moreover, B cells are positively associated with immunotherapy response in some tumors [36, 37]. Our study shows that B cells infiltration is positively correlated with TSHZ3 expression in STAD and BLCA. We also show that the expressions of some key marker for T cell exhaustion [38-40], such as PD-1(PDCD1) and TIM-3(HAVCR2), have positive correlations with TSHZ3 expression in STAD, suggesting that TSHZ3 plays a key role in T cell exhaustions mediated by PD-1 and TIM-3.

As for DCs infiltration, recent studies documented the biological functions of pDCs and cDC1s in tumors. pDCs, present the phenotypes as CD303(CLEC4C), CD304(NRP1), CD123(IL3RA), CCR2 and CXCR3, which predict poor prognosis of cancers by promoting the expansion of Treg cell populations $[12,41]$. CDC1s, present the phenotypes as HLA-DR, CD141, XCR1, CLEC9A and DEC-205(LY75), are often related to superior crosspresentation of antigens, which results in stronger CD8 $+\mathrm{T}$ cell immunity, and can additionally support Th1 cell polarization of CD4 + T cells [42-46]. These findings support our findings that pDCs infiltration has positive correlation with TSHZ3 expression level in STAD and BLCA.

NK cells are anti-tumorigenic TIICs [47]. However, our data show that NK cell infiltration has no significant correlation with TSHZ3 expression in STAD and BLCAThe infiltrations of Th17 cells, Tfh cells and neutrophils also have no significant correlations with TSHZ3 expression in STAD and BLCA. The effects of Th17 cells on cancers are bidirectional. On the one hand, Th17 cells promote tumor growth by secreting angiogenic factors (via IL-17) and exhibiting their immunosuppressive functions. On the other hand, Th17 cells also drive antitumor immune responses via recruiting immune cells, activating effector T cells (CD8+), or directly converting their phenotypes toward Th1 cells [48]. It could be a good explanation for our finding that the

Page $11 / 18$ 
secretion of IL-17A is negatively correlated with TSHZ3 expression in STAD and BLCA. High Tfh cells infiltrations have been reported to be related to good prognosis of some cancers [49]. Our study does not find a significant correlation between Tfh cells infiltration and TSHZ3 expression. Some studies reported that neutrophils are existed in different subsets which have diverse effects on cancers, but their phenotypic characteristics are less studied [50]. The correlation between the phenotypic characteristics of different neutrophil subsets and the TSHZ3 expression deserves further investigation. There are some limitations in our study. Firstly, we failed to divide some TIICs into different subsets for further evaluations because there is a lack of well-recognized phenotypic characteristics for these TIICs subsets. Secondly, though we enlarge the sample size in the meta-analysis with the Oncomine database, heterogeneity among the included studies may exist.

\section{Conclusion}

Overall, our study demonstrates that TSHZ3 expression is elevated in STAD and BLCA, which predicts the poor prognosis of the STAD and BLCA patients. Furthermore, TSHZ3 expression potentially contributes to the increased tumor infiltrations of M2 macrophages, pDCs, monocytes and B cells in STAD and BLCA, and TAMs, Th2 cells, Tregs and T cell exhaustion in STAD. Our findings strongly suggest that TSHZ3 plays a critical role in the immune infiltration and can be used as a prognostic biomarker in patients with STAD and BLCA.

\section{Declarations}

\section{Competing interests}

All authors declare that no conflict of interest exists regarding the submission of this work.

\section{Acknowledgements}

The authors are grateful to the help of Zhong-kuan Lyu in formation of figure.

\section{Availability of data and materials}

All the dataset supporting our findings was retrieved by using the Oncomine database, [https://www.oncomine.org/resource/login.html], GEPIA, [http://gepia.cancer-pku.cn/], Kaplan-Meier plotter, [http://kmplot.com/analysis/] and TIMER, [https://cistrome.shinyapps.io/timer/].

\section{Author's contributions}

Xi Zhang and Xiao-shan Zhao designed the study and interpreted data. Xi Zhang collected and analysed data, formed figures and wrote the manuscript. Min Wang, Han-qi Lu, Qiu-xing He, Yan-ting You, Xing-hong Zhou, Xiu-qiong Fu, Dong-hui Liang, Hiu Yee Kwan and Lin Zhou revised the manuscript.

\section{Ethics approval}

This study is not concerned with any clinical trials or animal experiments.

\section{Consent for publication}

Not applicable.

\section{Funding}

This work was supported by the Key Project of National Natural Science Foundation of China (No. 81830117) and the National Natural Science Foundation of China (No. 81673840).

\section{References}

1. Ferlay J, Soerjomataram I, Dikshit R, Eser S, Mathers C, Rebelo M, et al. Cancer incidence and mortality worldwide: sources, methods and major patterns in GLOBOCAN 2012. Int J Cancer 2015; 136: E359-86.

2. Cumberbatch M, Jubber I, Black PC, Esperto F, Figueroa JD, Kamat AM, et al. Epidemiology of Bladder Cancer: A Systematic Review and Contemporary Update of Risk Factors in 2018. Eur Urol 2018;7 4: 784-95. 
3. Matuszewski M, Szymanska B, Dlugosz A, Malkiewicz B, Dembowski J, Piwowar A. Preliminary Evaluation of the Diagnostic Usefulness of Uroplakin 2 with an Assessment of the Antioxidant Potential of Patients with Bladder Cancer. Biomed Res Int 2018; $2018: 8693297$.

4. Angell HK, Lee J, Kim KM, Kim K, Kim ST, Park SH, et al. PD-L1 and immune infiltrates are differentially expressed in distinct subgroups of gastric cancer. Oncoimmunology 2019; 8: e1544442.

5. Pan S, Zhan Y, Chen X, Wu B, Liu B. Bladder Cancer Exhibiting High Immune Infiltration Shows the Lowest Response Rate to Immune Checkpoint Inhibitors. Front Oncol 2019; 9: 1101.

6. Annels NE, Mansfield D, Arif M, Ballesteros-Merino C, Simpson GR, Denyer M, et al. Phase I Trial of an ICAM-1-Targeted ImmunotherapeuticCoxsackievirus A21 (CVA21) as an Oncolytic Agent Against Non Muscle-Invasive Bladder Cancer. Clin Cancer Res 2019; 25: 5818-31.

7. Solinas C, Pusole G, Demurtas L, Puzzoni M, Mascia R, Morgan G, et al. Tumor infiltrating lymphocytes in gastrointestinal tumors: Controversies and future clinical implications. Crit Rev Oncol Hematol 2017; 110: 106-16.

8. Lu J, Xu Y, Wu Y, Huang XY, Xie JW, Wang JB, et al. Tumor-infiltrating CD8+ T cells combined with tumor-associated CD68+ macrophages predict postoperative prognosis and adjuvant chemotherapy benefit in resected gastric cancer. Bmc Cancer 2019; $19: 920$.

9. Qi Y, Chang Y, Wang Z, Chen L, Kong Y, Zhang P, et al. Tumor-associated macrophages expressing galectin-9 identify immunoevasive subtype muscle-invasive bladder cancer with poor prognosis but favorable adjuvant chemotherapeutic response. Cancer Immunol Immunother 2019; 68: 2067-80.

10. Noh BJ, Kim JH, Eom DW. Prognostic Significance of Categorizing Gastric Carcinoma by PD-L1 Expression and Tumor Infiltrating Lymphocytes. Ann Clin Lab Sci 2018; 48: 695-706.

11. Ajili F, Kourda N, Darouiche A, Chebil M, Boubaker S. Prognostic value of tumor-associated macrophages count in human non-muscle-invasive bladder cancer treated by BCG immunotherapy. Ultrastruct Pathol 2013; 37: 56-61.

12. Wculek SK, Cueto FJ, Mujal AM, Melero I, Krummel MF, Sancho D. Dendritic cells in cancer immunology and immunotherapy. Nat Rev Immunol 2020; 20: 7-24.

13. Quail DF, Joyce JA. Microenvironmental regulation of tumor progression and metastasis. Nat Med 2013; 19: $1423-37$.

14. Chabbert D, Caubit X, Roubertoux PL, Carlier M, Habermann B, Jacq B, et al. Postnatal Tshz3 Deletion Drives Altered Corticostriatal Function and Autism Spectrum Disorder-like Behavior. Biol Psychiatry 2019; 86: 274-85.

15. Caubit X, Gubellini P, Andrieux J, Roubertoux PL, Metwaly M, Jacq B, et al. TSHZ3 deletion causes an autism syndrome and defects in cortical projection neurons. Nat Genet 2016; 48: 1359-69.

16. Caubit X, Lye CM, Martin E, Core N, Long DA, Vola C, et al. Teashirt 3 is necessary for ureteral smooth muscle differentiation downstream of SHH and BMP4. Development 2008; 135: 3301-10.

17. Li Y, Huang Y, Qi Z, Sun T, Zhou Y. MiR-338-5p Promotes Glioma Cell Invasion by Regulating TSHZ3 and MMP2. Cell Mol Neurobiol 2018; 38: 66977.

18. McBride DJ, Etemadmoghadam D, Cooke SL, Alsop K, George J, Butler A, et al. Tandem duplication of chromosomal segments is common in ovarian and breast cancer genomes. J Pathol 2012; 227: 446-55.

19. Natrajan R, Mackay A, Wilkerson PM, Lambros MB, Wetterskog D, Arnedos M, et al. Functional characterization of the $19 q 12$ amplicon in grade III breast cancers. Breast Cancer Res 2012; 14: R53.

20. Zeng H, Li H, Zhao Y, Chen L, Ma X. Transcripto-based network analysis reveals a model of gene activation in tongue squamous cell carcinomas. Head Neck 2019.

21. Elemeery MN, Badr AN, Mohamed MA, Ghareeb DA. Validation of a serum microRNA panel as biomarkers for early diagnosis of hepatocellular carcinoma post-hepatitis C infection in Egyptian patients. World J Gastroenterol 2017; 23: 3864-75.

22. Tang Z, Li C, Kang B, Gao G, Li C, Zhang Z. GEPIA: a web server for cancer and normal gene expression profiling and interactive analyses. Nucleic Acids Res 2017; 45: W98-102.

23. Li T, Fan J, Wang B, Traugh N, Chen Q, Liu JS, et al. TIMER: A Web Server for Comprehensive Analysis of Tumor-Infiltrating Immune Cells. Cancer Res 2017; 77: e108-10.

24. Rhodes DR, Kalyana-Sundaram S, Mahavisno V, Varambally R, Yu J, Briggs BB, et al. Oncomine 3.0: genes, pathways, and networks in a collection of 18,000 cancer gene expression profiles. Neoplasia 2007; 9: 166-80.

25. Li B, Severson E, Pignon JC, Zhao H, Li T, Novak J, et al. Comprehensive analyses of tumor immunity: implications for cancer immunotherapy. Genome Biol 2016; 17: 174.

26. Pan JH, Zhou H, Cooper L, Huang JL, Zhu SB, Zhao XX, et al. LAYN Is a Prognostic Biomarker and Correlated With Immune Infiltrates in Gastric and Colon Cancers. Front Immunol 2019; 10: 6.

27. Danaher P, Warren S, Dennis L, D'Amico L, White A, Disis ML, et al. Gene expression markers of Tumor Infiltrating Leukocytes. J Immunother Cancer 2017; 5: 18.

28. Aran D, Sirota M, Butte AJ. Systematic pan-cancer analysis of tumour purity. Nat Commun 2015; 6: 8971.

29. Japanese gastric cancer treatment guidelines 2014 (ver. 4). Gastric Cancer 2017; 20: 1-19.

Page $13 / 18$ 
30. Yoshihara K, Shahmoradgoli M, Martinez E, Vegesna R, Kim H, Torres-Garcia W, et al. Inferring tumour purity and stromal and immune cell admixture from expression data. Nat Commun 2013; 4: 2612.

31. Qian B, Deng Y, Im JH, Muschel RJ, Zou Y, Li J, et al. A distinct macrophage population mediates metastatic breast cancer cell extravasation, establishment and growth. Plos One 2009; 4: e6562.

32. Lin EY, Li JF, Gnatovskiy L, Deng Y, Zhu L, Grzesik DA, et al. Macrophages regulate the angiogenic switch in a mouse model of breast cancer. Cancer Res 2006; 66: 11238-46.

33. Gabrilovich DI, Nagaraj S. Myeloid-derived suppressor cells as regulators of the immune system. Nat Rev Immunol 2009; 9: 162-74.

34. Sasaki A, Iwashita Y, Shibata K, Matsumoto T, Ohta M, Kitano S. Prognostic value of preoperative peripheral blood monocyte count in patients with hepatocellular carcinoma. Surgery 2006; 139: 755-64.

35. de Visser KE, Korets LV, Coussens LM. De novo carcinogenesis promoted by chronic inflammation is B lymphocyte dependent. Cancer Cell 2005; 7 : 411-23.

36. Petitprez F, de Reynies A, Keung EZ, Chen TW, Sun CM, Calderaro J, et al. B cells are associated with survival and immunotherapy response in sarcoma. Nature 2020; 577: 556-60.

37. Helmink BA, Reddy SM, Gao J, Zhang S, Basar R, Thakur R, et al. B cells and tertiary lymphoid structures promote immunotherapy response. Nature 2020; 577: 549-55.

38. Huang YH, Zhu C, Kondo Y, Anderson AC, Gandhi A, Russell A, et al. CEACAM1 regulates TIM-3-mediated tolerance and exhaustion. Nature 2015; 517: 386-90.

39. Zhou Q, Munger ME, Veenstra RG, Weigel BJ, Hirashima M, Munn DH, et al. Coexpression of Tim-3 and PD-1 identifies a CD8+ T-cell exhaustion phenotype in mice with disseminated acute myelogenous leukemia. Blood 2011; 117: 4501-10.

40. Wherry EJ. T cell exhaustion. Nat Immunol 2011; 12: 492-9.

41. Aspord C, Leccia MT, Charles J, Plumas J. Plasmacytoid dendritic cells support melanoma progression by promoting Th2 and regulatory immunity through OX40L and ICOSL. Cancer Immunol Res 2013; 1: 402-15.

42. Wculek SK, Amores-Iniesta J, Conde-Garrosa R, Khouili SC, Melero I, Sancho D. Effective cancer immunotherapy by natural mouse conventional type-1 dendritic cells bearing dead tumor antigen. J Immunother Cancer 2019; 7: 100.

43. Salmon H, Idoyaga J, Rahman A, Leboeuf M, Remark R, Jordan S, et al. Expansion and Activation of CD103(+) Dendritic Cell Progenitors at the Tumor Site Enhances Tumor Responses to Therapeutic PD-L1 and BRAF Inhibition. Immunity 2016; 44: 924-38.

44. Sanchez-Paulete AR, Cueto FJ, Martinez-Lopez M, Labiano S, Morales-Kastresana A, Rodriguez-Ruiz ME, et al. Cancer Immunotherapy with Immunomodulatory Anti-CD137 and Anti-PD-1 Monoclonal Antibodies Requires BATF3-Dependent Dendritic Cells. Cancer Discov 2016; 6: 71-9.

45. Jongbloed SL, Kassianos AJ, McDonald KJ, Clark GJ, Ju X, Angel CE, et al. Human CD141+ (BDCA-3)+ dendritic cells (DCs) represent a unique myeloid DC subset that cross-presents necrotic cell antigens. J Exp Med 2010; 207: 1247-60.

46. Hildner K, Edelson BT, Purtha WE, Diamond M, Matsushita H, Kohyama M, et al. Batf3 deficiency reveals a critical role for CD8alpha+ dendritic cells in cytotoxic T cell immunity. Science 2008; 322: 1097-100.

47. Vivier E, Tomasello E, Baratin M, Walzer T, Ugolini S. Functions of natural killer cells. Nat Immunol 2008; 9: 503-10.

48. Guery L, Hugues S. Th17 Cell Plasticity and Functions in Cancer Immunity. Biomed Res Int 2015; 2015: 314620.

49. Gu-Trantien C, Migliori E, Buisseret L, de Wind A, Brohee S, Garaud S, et al. CXCL13-producing TFH cells link immune suppression and adaptive memory in human breast cancer. JCl Insight 2017; 2.

50. Fridlender ZG, Albelda SM. Tumor-associated neutrophils: friend or foe? Carcinogenesis 2012;33: 949-55.

\section{Figures}


Figure 1

Comparison of TSHZ3 Across 5 Analyses

Over-expression / Copy Number Gain

A

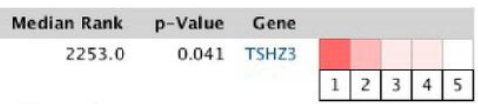

\begin{tabular}{|l|l|l|l|l|}
\hline 1 & 2 & 3 & 4 & 5 \\
\hline
\end{tabular}

Legend

1. Infiltrating Bladder Urothelial Carcinoma vs. 4. Infiltrating Bladder Urothelial Carcinoma vs.

Normal

Lee Bladder, J Clin Oncol, 2010

Normal.

2. Superficial Bladder Cancer vs. Normal $\quad 5$. Infiltrating Bladder Urothelial Carcinoma,

Lee Bladder, J Clin Oncol, $2010 \quad$ Micropapilary Variant vs. Normal.

3. Bladder Urothelial Carcinoma vs. Normal TCGA Blodder, No Associated Poper, 2013

TCGA Bladder, No Associated Paper, 2013

$\begin{array}{lllll}5 & 1025 & 25 & 10 & 5\end{array}$

ㅁㅁㅁㅁㅁ $\square$ Not measured

The rank for a gene is the median rank for that gene across each of the analyses
The p.Value for a gene is its p.value for the median-ranked analysis.

Comparison of TSHZ3 Across 17 Analyses

Over-expression / Copy Number Gain

Median Rank p-Value Gene

B
6957.0
0.006 TSHZ3

Legend

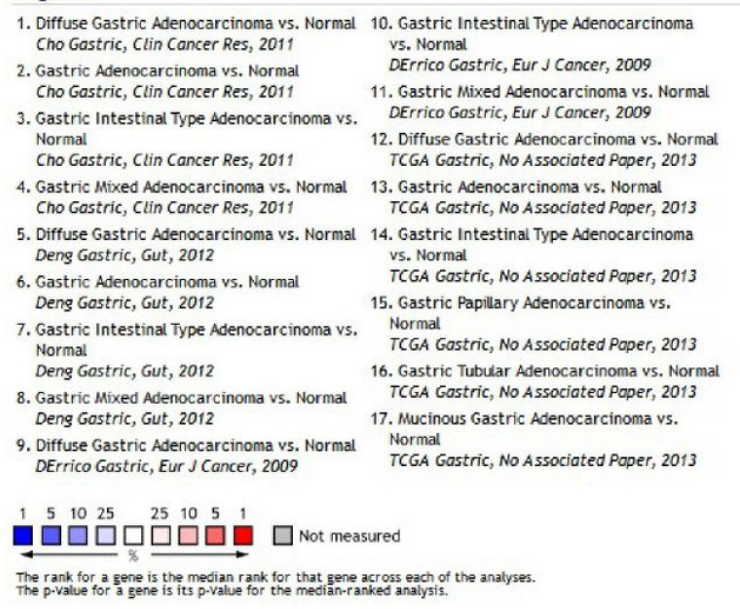

Figure 1

TSHZ3 expression in BLCA and STAD. (A) TSHZ3 expression in BLCA. (B) TSHZ3 expression in STAD. 
Figure 2

Al
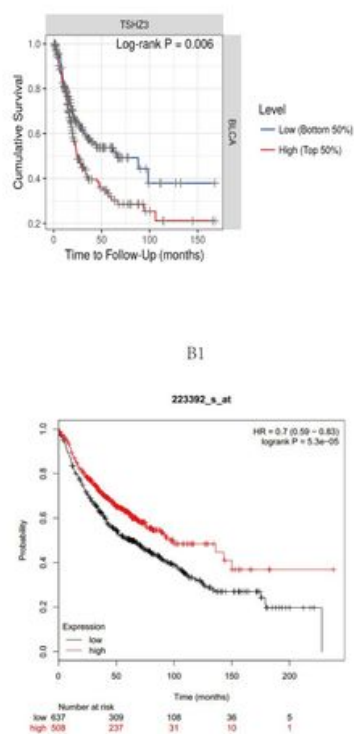

CI

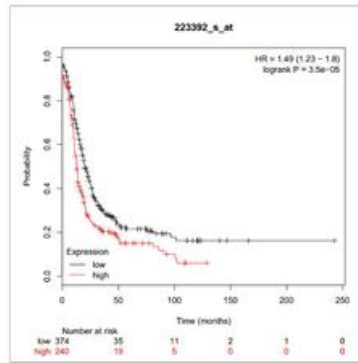

A2

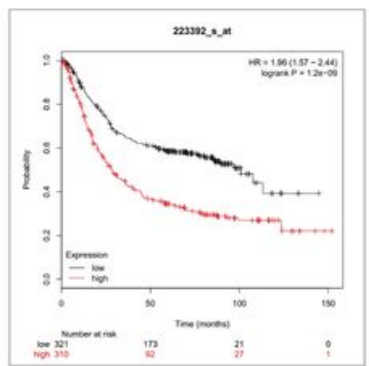

B2

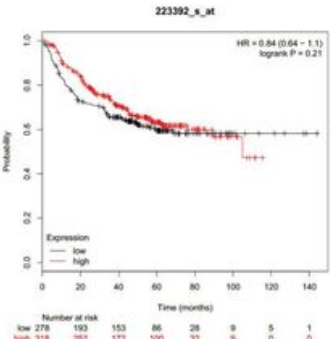

C2

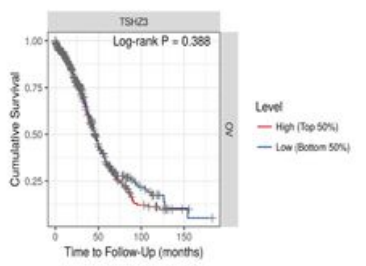

A3

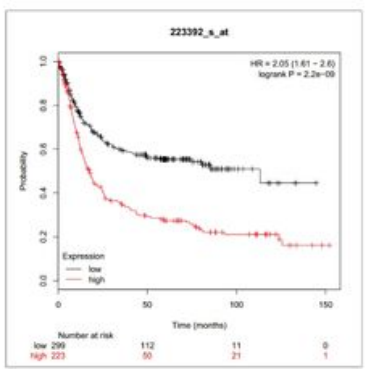

B3

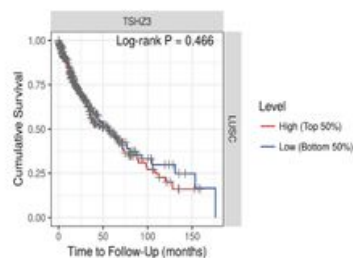

C3

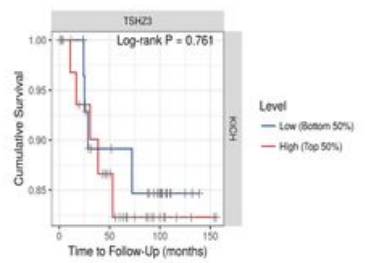

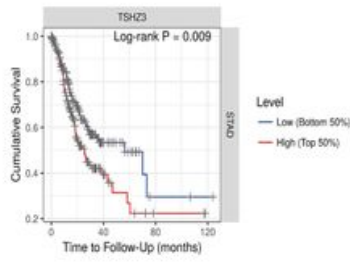

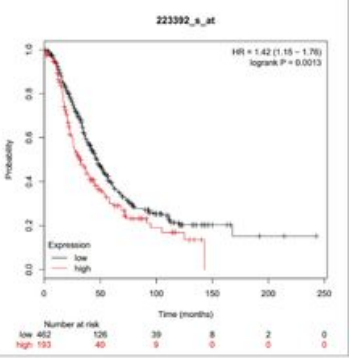

Figure 2

Kaplan-Meier plots comparing the high and low expression of TSHZ3 in cancers with TSHZ3 differential expression by Kaplan-Meier plotter (A2, A3, B1, B2, B4 and C1) and TIMER (A1, A4, B3, C2 and C3). (A1) Kaplan-Meier plot of OS in BLCA (bladder urothelial carcinoma) ( $n=406)$. (A2-A3) Kaplan-Meier plot of OS and PFS in gastric cancer ( $n=631, n=522)$. (A4) Kaplan-Meier plot of OS in STAD (stomach adenocarcinoma) ( $n=408)$. (B1-B2) Kaplan-Meier plot of OS and PFS in lung cancer $(n=1145, n=596)$. (B3) Kaplan-Meier plot of OS in LUSC (lung squamous cell carcinoma) ( $n=495)$. (B4-C1) KaplanMeier plot of OS and PFS in ovary cancer $(n=655, n=614)$. (C2) Kaplan-Meier plot of OS in OV (ovarian serous cystadenocarcinoma) ( $n=561)$. (C3) Kaplan-Meier plot of OS in KICH (Kidney Chromophobe) ( $n=65)$. OS, overall survival; PFS, progression-free survival.

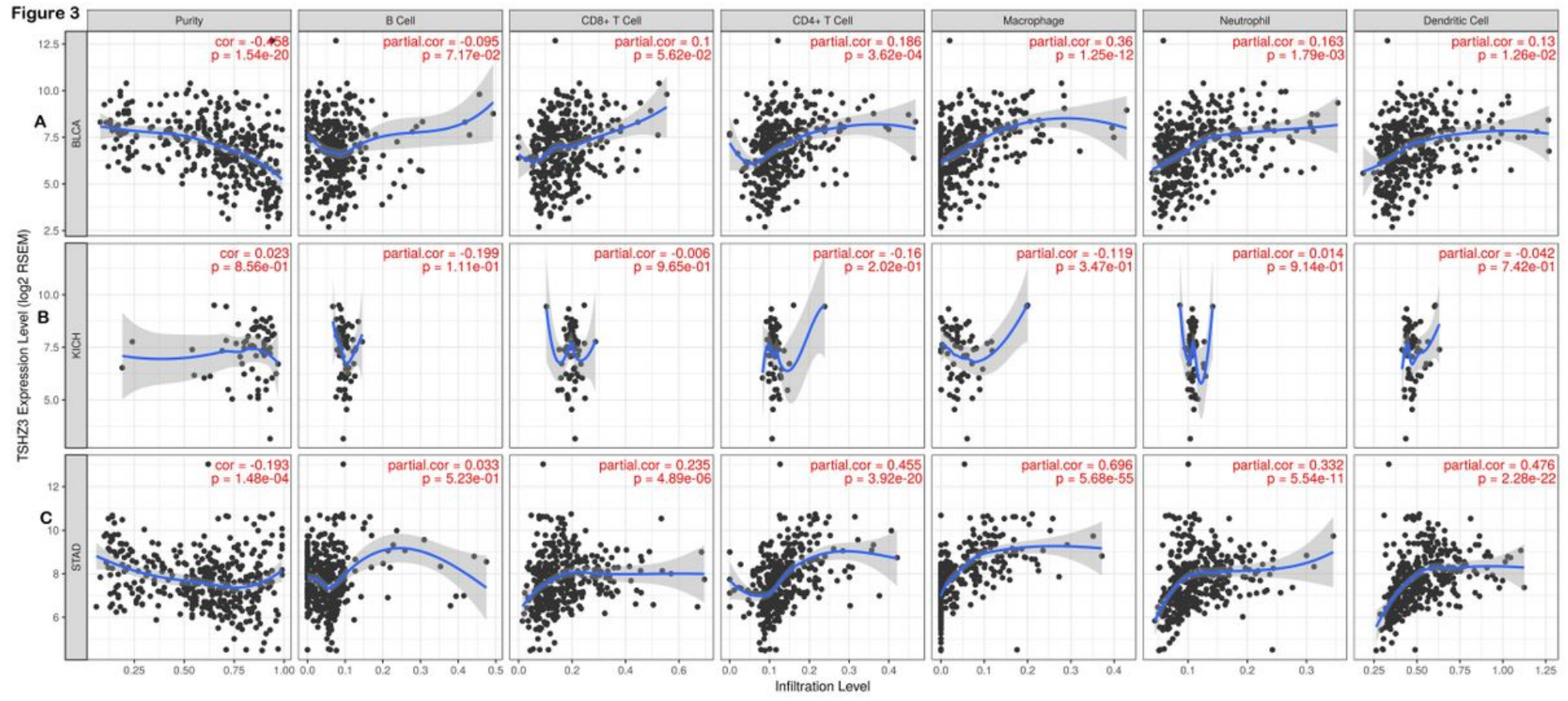


Figure 3

Rough evaluation reveal the correlation of immune infiltration level with TSHZ3 expression in BLCA, STAD and KICH. (A) TSHZ3 expression is significantly negatively correlated to tumor purity and has significant positive correlations with infiltrating levels of CD4+ T cells, macrophages, neutrophils, and dendritic cells but not B cells and CD8+ T cells in BLCA. $(n=408)$. (B) TSHZ3 expression has no significant correlations with tumor purity and infiltrating levels of B cells, CD8+ T cells, CD4+ T cells, macrophages, neutrophils, and dendritic cells in KICH ( $n=66)$. (C) TSHZ3 expression is significantly negatively related to tumor purity and has significant positive correlations with infiltrating levels of CD8+ T cells, CD4+ T cells, macrophages, neutrophils, and dendritic cells but not B cells in STAD $(n=415)$. BLCA (colon adenocarcinoma), STAD (stomach adenocarcinoma) and $\mathrm{KICH}$ (kidney chromophobe).

Figure 4

A

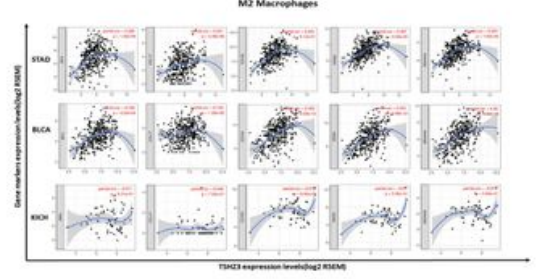

noc

C

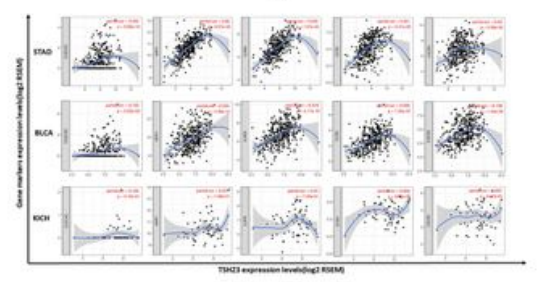

Treg

E

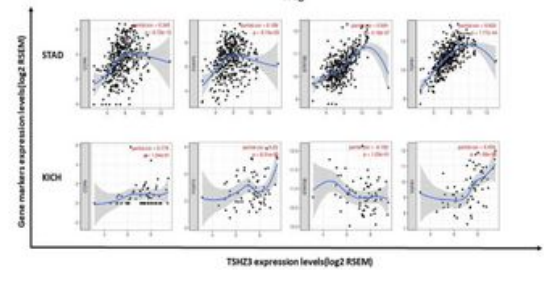

Bcell

G
B

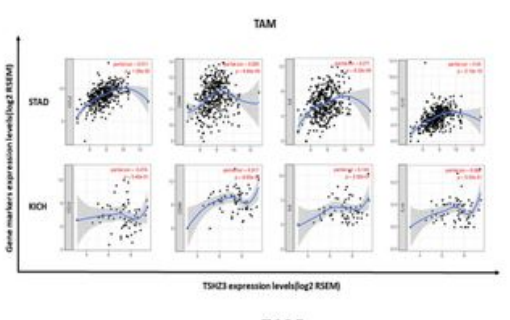

D

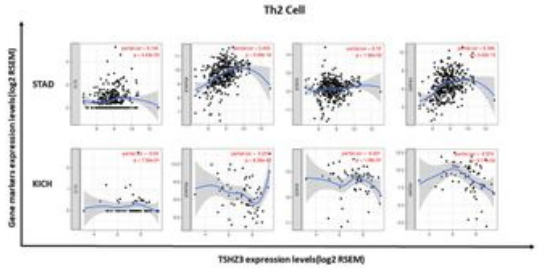

F

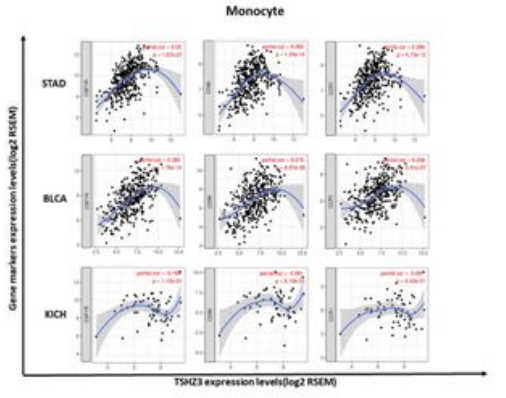

Figure 4

Refined analysis on the correlation between TSHZ3 Expression and Immune Infiltrates in BLCA, STAD and KICH. (A) M2 macrophages infiltration in STAD, BLCA and $\mathrm{KICH}$. (B) TAMs infiltration in STAD and $\mathrm{KICH}$. (C) pDCs infiltration in STAD, BLCA and KICH. (D) Th2 cells infiltration in STAD and $\mathrm{KICH}$. (E) Tregs infiltration in STAD and KICH. (F) Monocytes infiltration in STAD, BLCA and KICH. (G) B cells infiltration in STAD, BLCA and KICH. STAD (stomach adenocarcinoma), BLCA (colon adenocarcinoma), $\mathrm{KICH}$ (kidney chromophobe).

\section{Supplementary Files}

This is a list of supplementary files associated with this preprint. Click to download.

- SupplementaryTableS1.docx

- SupplementaryFigureS1.jpg

- SupplementaryFigureS2.jpg

- SupplementaryFigureS1.jpg

- SupplementaryFigureS2.jpg

- SupplementaryTableS1.docx 
- SupplementaryTableS2.docx

- SupplementaryTableS2.docx

Page 18/18 